\title{
Study on the Home Health Caregiver Scheduling Problem under a Resource Sharing Mode considering Differences in Working Time and Customer Satisfaction
}

\author{
Heping Wang, Yuke He, Yan Li ${ }^{(D}$, and Fuyu Wang \\ School of Management Science and Engineering, Anhui University of Technology, Maanshan 243032, China \\ Correspondence should be addressed to Yan Li; liyan4@ahut.edu.cn
}

Received 3 February 2020; Accepted 18 March 2020; Published 11 May 2020

Guest Editor: Jinliang Wang

Copyright (c) 2020 Heping Wang et al. This is an open access article distributed under the Creative Commons Attribution License, which permits unrestricted use, distribution, and reproduction in any medium, provided the original work is properly cited.

\begin{abstract}
The stage of social aging and further deepening of population aging has been witnessed in China. The demand for home care is increasingly growing; meanwhile, medical human resources are insufficient. In this context, a home health caregiver scheduling problem under the resource sharing mode is studied in this paper. Under such mode, two types of caregivers, i.e., full-time caregiver and part-time caregiver, are regarded as the main labor force by HHC institutions. In HHC planning, different working times for the two kinds of caregivers will need to be considered. Consequently, in this paper, a corresponding mathematical model is established and a hybrid algorithm that combines the whale optimization algorithm (WOA) with the particle swarm optimization (PSO) algorithm is proposed to solve the model. The proposed algorithm is compared with the existing algorithms to verify its effectiveness through three example tests of different scales and Solomon example. Finally, the resource sharing model is compared with the traditional model through a case, and the rationality of home health caregiver scheduling in the resource sharing model is discussed in terms of cost structure and customer satisfaction.
\end{abstract}

\section{Introduction}

The stage of social aging and further deepening of population aging has been witnessed in China. The problem of old-age care is getting more and more serious. The existing traditional pension model has not been able to meet the needs of the elderly well. A novel home health care (HHC) model has been developed and extensively applied in both Europe and North America [1]. This model has been gradually accepted and recognized by an increasing number of people with the growth of the Chinese economy, the advance of science and technology, and the improvement of both the living standard and lifestyle of people in China. Home health care in China is mainly implemented by two entities: community-based well-known local hospitals and home-based medical care companies. According to the China Health Statistics Yearbook 2018, only 1.8 medical technicians care for every 1000 people; consequently, there is a significant shortage in medical resources
[2]. For the newly emerging home health care industry in China, nursing staff has become the most scarce resource. The hospital has a large number of health caregivers, and the health caregivers have some spare time under the scheduling system of hospital. The home health care industry can use the resource sharing model to solve the resource shortage under the background of the decentralization of idle resources, which can effectively develop the family medical care industry and activate the flexibility of employment in the medical industry. Under the resource sharing mode, HHC institutions employ part-time health caregivers to support daily home health caregiver scheduling. After accepting employment by a well-known community-based local hospital and a home-based medical care company, medical health caregivers must balance both jobs according to their respective working schedules. Given that medical institutions have different schedules, HHC medical health caregivers have different working times. Thus, when designing routing and scheduling plans, HHC 
centers must consider the different working times of health caregivers.

Scholars have conducted a series of studies on home health caregiver scheduling. Redjem et al. [3] proposed two integer programming models; each model corresponded to a specific home health caregiver scheduling strategy. They conducted experiments that identified a restrictive relationship between customer waiting time and health caregiver travel time. Rasmussen et al. [4] introduced a constrained set partitioning model to summarize the travel time for visiting different customers and the visit time window. This problem was solved using the B\&P algorithm. Numerical simulation indicated that the result was very close to the optimal solution. Larsen et al. [5] modeled HCSP as a multistage (multilevel) combinatorial optimization problem. They developed a modified version for practical problem solving using a tabu search-based heuristic algorithm. Braekers et al. [6] proposed a metaheuristic algorithm, with a large neighborhood search algorithm added to a multiple local search box forward. Tricoire et al. [7] emphasized the need to estimate health caregiver travel time when solving a scheduling problem using the two-stage method. They estimated the travel time using a kernel regression algorithm based on health caregiver travel times observed during previous periods. The numerical calculation on the basis of the examples practical problems shows that the estimation effect of this method is better than that of the classical mean value method.

Liu et al. [8] considered the lunch time of health caregivers in home health caregiver scheduling and built a threeindex mathematical model to solve the problem. They decomposed the problem into one master problem and several pricing subproblems and used the branch-and-price algorithm to find the optimal solution. Zhuo et al. [9] proposed a tabu search algorithm based on insertion algorithm and saving algorithm to solve the management problem of home-based medical and health care resources with cyclical service demands. To solve the home health caregiver scheduling problem of simultaneous services, Yang-yi et al. [10] designed an adaptive large neighborhood search algorithm in which multiple removing and insertion algorithms were used for a neighborhood search in feasible and infeasible solution spaces. It adopted a relation matrix and time adjustment strategies to accelerate problem solving and applied a simulated annealing method to accept neighborhood solutions. Finally, numerical experiments and algorithm comparison were carried out to verify the effectiveness of algorithm. Yuan et al. [11] proposed a mathematical model with constraints of multiple types of caregivers and converted it into a major problem based on set partitioning and several pricing subproblems based on the shortest path with resource constraints. Each of these paths corresponds to one type of health caregiver. A column generation algorithm and $\mathrm{B} \& \mathrm{P}$ algorithm were designed to solve the model. Fang et al. [12] considered the problem of finite-time stabilization in probability for a class of highorder stochastic nonlinear systems with output constraints in which the fractional powers are only required to be positive rather than not less than one. Zhao et al. [13] considered the second-order sliding mode controller design subject to an output constraint. By constructing a new barrier Lyapunov function and applying the technique of adding a power integrator, a novel second-order sliding mode control algorithm, which can be used to deal with the output constraint problem, has been developed. Liang et al. [14] proposed the problem of dissipativity analysis and nonfragile sampled-data control which is investigated for fuzzy Markovian jump systems, and based on the mode-dependent Lyapunov function consisting of a two-sided closed-loop function, the stochastically stable criteria, and strictly dissipative criteria, a truck-trailer model is given to demonstrate the efficiency of the presented method. Xia et al. [15] proposed a command filtered-based adaptive fuzzy tracking control scheme for nonlinear systems with unknown control directions. Combining fuzzy logic system and command filter with Nussbaum gain function, adaptive fuzzy controller constructed in this paper guarantees that error signals converge into bounded compact sets around the origin and all closed-loop signals are bounded. The effectiveness of the presented scheme is verified by a simulation result.

Due to the flexibility of the resource sharing model, many scholars have solved many industrial problems by using the resource sharing model. Yan-ning et al. [16] designed the optimization problem of vehicle logistics path under the resource sharing mode and constructed the mathematical model of path optimization. AlQahtani [17] proposed a resource sharing scheme to solve the data traffic congestion and overload in 5G network. Wu et al. [18] proposed a model of sharing manufacturing resources to solve the decentralization, complexity, and heterogeneity of manufacturing resources. Wu et al. [19] studied the green resource sharing problem of mobile device-to-device (D2D) based on cellular network and established an energy efficiency analysis model.

From the above literatures, many scholars have studied models and algorithms of home health care resources scheduling and have obtained some achievements. There have been many complex models that approximate a real problem. Also, some effective intelligent optimization algorithms and exact solution algorithm have been presented. However, these existing studies have focused on full-time health caregiver scheduling. Few scholars consider both fulltime and part-time health caregivers' scheduling. Actually due to shortages in Chinese medical resources, many home health care centers need to hire part-time health caregivers to meet nursing demand of older customers. This problem is becoming increasingly widespread and persistent. To resolve the supply-demand contradiction in health care resources based on the above studies, this paper studies the home health care scheduling problem in resource sharing mode.

The main contributions of the paper include the following points: (1) we propose a home health care scheduling model considering customer satisfaction under a resource sharing mode; (2) in the model, the different working time windows of health caregivers are considered; (3) a hybrid algorithm of whale optimization algorithm (WOA) and particle swarm optimization (PSO) (i.e., WOA-PSO) is designed. 
The paper is organized as follows. A mathematical model of home health caregiver scheduling problem is presented in Section 2. A hybrid intelligent optimization algorithm is designed in Section 3. Numerical experiments are conducted in Section 4. Conclusion is given in Section 5.

\section{Modeling}

2.1. Problem Description. This paper studies the home health care scheduling in resource sharing mode. In this mode, if the full-time health caregivers cannot meet the needs of the elderly customers, home health care center will dispatch part-time staff to serve the elderly customers. The difference between the new service route network and the traditional one is shown in Figure 1.

The problem posed in this paper can be described on a directed graph $G(V, A)$, where $V$ denotes a set of nodes $V=\{0,1,2, \ldots, N\}$, node 0 represents the HHC center, and nodes $1,2, \ldots, N$ signify customers. $A$ signifies a set of edges; $A=\{(i, j) \mid i, j \in V, i \neq j\}$. Each customer has a definite request and service time. The service time of node 0 is 0 . There are many health caregivers in HHC center. Each customer has an expected time window and a tolerable time window. Each health caregiver has a working time window. When the working time window is open, the health caregiver leaves the health care center to provide customer service. The home caregiver has to wait until the time window opens if the health caregiver arrives at the customer before the start of the time window. If the health caregiver arrives later than the tolerable time window, the customer will refuse his or her service. The arrival time of health caregiver is their starting service time. The health caregivers will continue to serve the next customer when their service time exceeds the working time window, which will result in service cost and overtime penalty cost. The health caregiver does not have to return to the health care center.

In order to formulate the home health caregiver scheduling problem, all symbols for the model are as shown in the following:

$i, j$ : HHC center if $i, j=0$ and customer if $i, j \neq 0$

$k$ : health caregiver

$V$ : a set of customers and the HHC center

$H$ : a set of health caregivers

$S_{i k}$ : service time of customer $i$ by health caregiver $k$

$\left[E_{i}, L_{i}\right]:$ an expected time window of customer $i$

$\left[E E_{i}, L L_{i}\right]$ : a tolerable time window of customer $i$

$t_{i j}$ : the travel time between customer $i$ and customer $j$

$c_{1}$ : the unit travel cost of each health caregiver

$c_{2}$ : the unit service cost of each health caregiver

$c_{3}$ : the unit overtime cost of each health caregiver

$c_{4}$ : the unit waiting time cost of each health caregiver $\left[W S_{k} \cdot W E_{k}\right]$ : working time window for health caregiver $k$

$A T_{i k}$ : the starting time of health caregiver $k$ serving customer $i$ $t_{k}$ : the off-duty time of health caregiver $k$

$M$ : big positive real number

$x_{i j k}: 0-1$ binary variable, 1 if health caregiver $k$ travels from $i$ to $j$ for providing service, 0 otherwise.

2.2. Satisfaction Function. Customers have different tolerances time windows and expected time windows, so the starting time of health care directly influences customer satisfaction. A shorter interval between the starting time of health care and the expected time window leads to higher customer satisfaction. If the starting time is within the expected time window, the customer is most satisfied and satisfaction value is 1 . Considering that different customers have different perceptions of time, a time sensitivity coefficient $\delta$ is established in this paper. The satisfaction function is as follows:

$$
F\left(A T_{i K}\right)= \begin{cases}\left(\frac{A T_{i k}-E E_{i}}{E_{i}-E E_{i}}\right)^{\delta}, & E_{i}>A T_{i k}>E E_{i} \\ 1, & L_{i} \geq A T_{i k} \geq E_{i}, \\ \left(\frac{A T_{i k}-L_{i}}{L L_{i}-L_{i}}\right)^{\delta}, & L L_{i}>A T_{i k}>L_{i}, \\ 0, & \text { others. }\end{cases}
$$

2.3. Home Health Caregiver Scheduling Model. The optimization objective of dispatching health caregivers is to minimize the health care center's total serving cost and maximize customer satisfaction.

Based on the above description and mathematical symbols, the mathematical model for the problem is

$$
\begin{aligned}
\max f_{1}= & \sum_{i \in V} \sum_{k \in H} F\left(A T_{i k}\right), \\
\min f_{2}= & \sum_{i \in V} \sum_{j \in V} \sum_{k \in H} x_{i j} t_{i j} c_{1}+\sum_{k \in H} \sum_{i \in V} c_{2} S_{i k} \\
& +\sum_{k \in H} c_{3} \max \left(t_{k}-W E_{k}, 0\right) \\
& +\sum_{k \in H} \sum_{i \in V} \sum_{j \in V} c_{4} \max \left(E E_{i}-A T_{i j}, 0\right) x_{i j k},
\end{aligned}
$$

subject to

$$
\begin{aligned}
\sum_{k \in H} \sum_{i \in V} x_{i j k} & =1, \quad \forall j \in V, \\
\sum_{j \in V} \sum_{k \in H} x_{i j k} & =1, \quad \forall i \in V, \\
\sum_{j \in V} x_{0 j k} & =1, \quad \forall k \in H,
\end{aligned}
$$



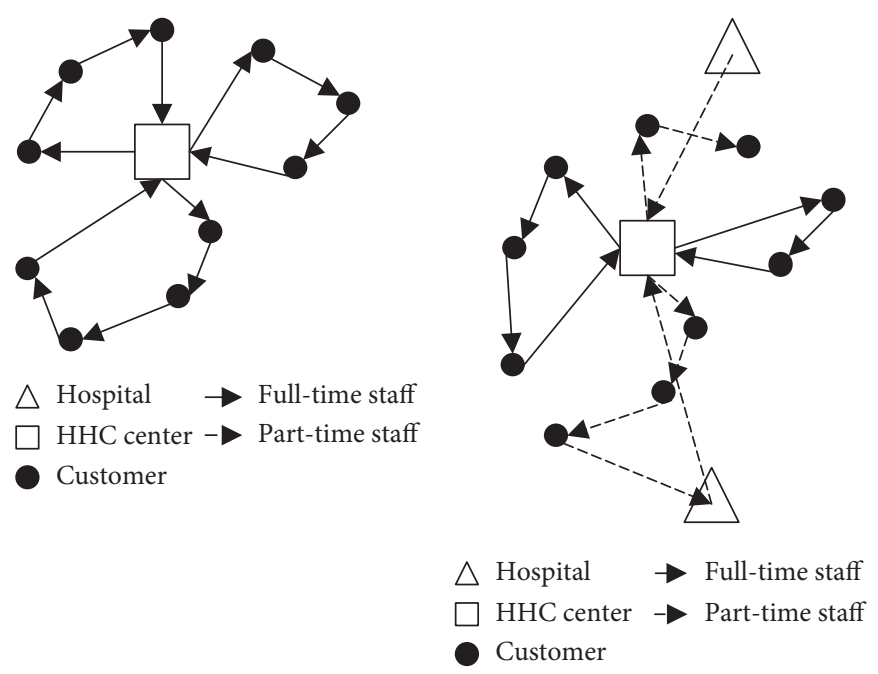

(a)

(b)

Figure 1: Schematic diagram of (a) traditional mode and (b) resource sharing mode.

$$
\begin{gathered}
\sum_{i \in V} x_{i o k}=1, \quad \forall k \in H, \\
\sum_{i \in V} x_{i j k}=\sum_{i \in V} x_{j i k}, \quad \forall k \in H, \forall j \in V, \\
A T_{j k}+M\left(1-x_{i j k}\right) \geq A T_{i k}+S_{i k}+t_{i j}, \quad \forall i \in V, \forall j \in V, \\
\forall k \in H,
\end{gathered}
$$

$$
E E_{i} \leq A T_{i k} \leq L L_{i}, \quad \forall \mathrm{i} \in V, \forall k \in H
$$

Objective function formula (2) represents the maximization of customer satisfaction; objective function formula (3) represents the minimization of health care center's total serving cost, including traveling cost, service cost, overtime cost, and waiting cost; constraint equations (4) and (5) ensure that each customer is served by only one health caregiver; constraint equations (6) and (7) ensure that each service route is serviced by only one health caregiver; constraint equation (8) ensures continuity of service; constraint equation (9) ensures the time sequence of service; constraint equation (10) ensures that the starting time of the service cannot exceed the customer's latest tolerable time window.

2.4. Model Processing. The proposed HHC scheduling optimization model in this study includes two objectives: maximizing customer satisfaction and minimizing health care center's serving total cost. This model is a multiobjective optimization model. Available methods to solve multiobjective optimization models include the weight coefficient method and the Pareto optimality method. The weight coefficient method has higher feasibility and lower time complexity. Thus, in this study, it is adopted for model processing. However, it cannot be weighted directly because the model has the dual objectives of maximizing customer satisfaction and minimizing $\mathrm{HHC}$ costs. Therefore, the following measures are taken:

(1) By taking the reciprocal of customer satisfaction, the maximum of the customer satisfaction is converted into the minimum of the reciprocal of customer satisfaction. Then, it is weighted with the health care center's total serving cost.

(2) The conversion of customer satisfaction into its reciprocal will yield a difference of several orders of magnitude between cost and the value of the satisfaction function. Thus, the reciprocal of customer satisfaction is multiplied by an equivalence factor to support the equalization and dimensionless of the two objectives.

Based on the above processing, the objective function of this model is

$$
\begin{aligned}
& \min \frac{f_{2}=\rho_{1} \beta}{\sum_{i \in N} \sum_{k \in K} F\left(A T_{i k}\right)}+\rho_{2}\left[\sum_{i \in V} \sum_{j \in V} \sum_{k \in H} x_{i j} t_{i j} c_{1}+\sum_{i \in V} \sum_{k \in H} c_{2} S_{i k}\right. \\
& \left.+\sum_{k \in H} \max \left(t_{k}-W E_{k}, 0\right) c_{3}+\sum_{i \in V} \sum_{j \in V} \sum_{k \in H} \max \left(E E_{j}-A T_{i j}, 0\right) x_{i j k} c_{4}\right]
\end{aligned}
$$

\section{A Hybrid WOA-PSO Algorithm}

3.1. Standard WOA. WOA was first proposed by Mirjalili [20] in 2016. It is a novel metaheuristic algorithm that is derived by simulating the behavior that the individual whales communicate with each other through ultrasound when whale swarms hunt for prey or conduct other group activities. When a whale discovers prey, it sends ultrasonic signals to inform its companions about the prey. When hunting, each individual in a whale swarm receives 
considerable information from other individuals through ultrasound and approaches the prey based on this information.

The search mode in the WOA is divided into a local development stage and a global search stage.

During the global search stage, a stochastic search agent is selected and defined as the current optimal candidate solution. Other search agents are considered to be far from this optimal candidate solution. The mathematical description is as follows:

$$
\begin{aligned}
D & =\left|\vec{C} \cdot t \vec{X}^{*} n(t) q-h \vec{X}(t)\right|, \\
\vec{X}(t+1) & =\vec{X}^{*}(t)-\vec{A} \cdot \vec{D},
\end{aligned}
$$

where $t$ denotes the current number of iterations; $\vec{A}$ and $\vec{C}$ denote coefficient vectors; $\vec{X}^{*}(t)$ is the current optimal position vector, which requires updates for each generation; $\vec{X}(t)$ is the position vector; is the absolute value; and $\cdot$ is an element-by-element multiplication. $\vec{X}^{*}(t)$ will be updated in each iteration if there is a better solution.

The vectors $\vec{A}$ and $\vec{C}$ are calculated as follows:

$$
\begin{aligned}
& \vec{A}=2 \vec{\alpha} \cdot \vec{r}-\vec{\alpha}, \\
& \vec{C}=2 \cdot \vec{r}, \\
& \vec{\alpha}=2-\frac{2 t}{I_{\text {gen } \max }},
\end{aligned}
$$

where $\vec{r}$ is a stochastic vector in $[0,1]$ and $I_{\text {gen } \max }$ is the set maximum number of iterations; $\vec{\alpha}$ is linearly decreased from 2 to 0 over the course of iterations. When a "subswarm" approaches the optimal candidate position in the whale swarm, $\vec{A}$ is stochastically selected within the range $[-2,-1] \cup[1,2]$ to keep other "subswarms" away from the optimal candidate position and avoid being caught in local optimization.

During the local development stage, WOA describes the bubble-net behavior through shrinking encircling and spiral updating position. The spiral updating position is a logarithm spiral curve between the current position and the optimal position. Its mathematical expression is as follows:

$$
\vec{X}(t+1)=\vec{D}_{1} \cdot e^{h l} \cdot \cos (2 \pi l)+\vec{X}^{*}(t),
$$

where $D=X * t-X t ; h$ is a constant for defining the spiral shape; and $l$ is a stochastic number within the range of $[-1,1]$.

The modes of shrinking encircling and spiral updating position proceed simultaneously. The probability factor $\rho$ determines the mode used by an agent for local development, where $\rho$ denotes a stochastic number within the range of $[0,1]$.

$$
\vec{X}(t+1)= \begin{cases}\vec{X}^{*}(t)-\vec{A} \cdot \vec{D}, & \rho<0.5, \\ \vec{D}_{1} \cdot e^{h l} \cdot \cos (2 \pi l)+\vec{X}^{*}(t), & \rho \geq 0.5 .\end{cases}
$$

Random numbers such as $\vec{\alpha}, \vec{A}, \vec{r}$, and $\rho$ make the algorithm more accidental during search. More importantly, $\vec{\alpha}$ makes the algorithm transition to local search slowly as the number of iterations increases, which results in the second half of the algorithm falling into local optimum. Considering the excellent performance of the WOA to solve multiobjective nonlinear optimization problems, a hybrid mechanism is introduced to overcome the defect of the algorithm and to further effectively optimize the solutions in this paper.

\subsection{Hybrid Algorithm Based on WOA and PSO}

3.2.1. Algorithm Principles. WOA is used for global optimization in the hybrid algorithm so that solution spaces gather in the area of the global optimal solution. Based on WOA, the fitness values of individuals can be calculated and a historically optimal value is recorded. Then, the learning mechanism of PSO algorithm is introduced to learn from historically optimal individuals and conduct screening. The learning mechanism enhances the local search ability of "subswarms" far away from the optimal candidate solution. During the later stage of the algorithm, when the swarm has been caught in the local optimization, individuals trace the current optimal solution through the learning mechanism and search the local new solutions, which make the algorithm jump out of the local optimization more easily. The mathematical expression of the learning mechanism of PSO is

$$
L_{n+1}=L_{n}+M_{1} * \operatorname{rand}(0,1) *\left(L_{n}-L_{\text {best }}\right),
$$

where the greater the difference between an individual and the historic optimized value, the bigger the learning factor $M_{1}$.

A league competition mechanism is also introduced into the algorithm to enhance the global search ability. The league competition mechanism replaces the stochastic selection in the standard WOA. First, a specific number of individuals are selected from the population with equal probability. Then, the best individual is selected among them and implanted into the progeny population until the new population scale reaches the original population scale.

The specific steps are as follows:

(1) Determine the number of individuals to be selected

(2) Calculate the fitness value of each individual and select the individual with the best fitness value for direct admission into the next generation

(3) Repeat step (2) until the new population scale reaches the original population scale

3.2.2. Problem-Based Coding Scheme. The WOA-PSO algorithm is used to solve multiobjective combinatorial optimization problems. An individual in each search space represents a solution to the space. The home health caregiver scheduling problem is a NP-hard problem that requires solving both the service sequence and routing of health caregivers. Thus, service selection and route sequence should 
be mapped onto each individual. The position vector of each individual is an $\mathrm{N}$-dimensional real vector. The integral part in the vector denotes the serial number for the health caregiver who should serve the $i$ th customer, while the decimal part denotes the route of the health caregiver. Assuming that eight customers and three health caregivers work in an area, Figure 2 shows the position vector of an individual.

When $[X]$ denotes the integer part of each gene locus for an individual, the same integer value indicates that the customer is served by the same health caregiver. When $\{X\}$ denotes the decimal part, a greater decimal value means a more forward position in the route sequence (see decoding steps in Figure 3). Thus, the scheme of the individual can be described as follows: customers 2, 4, and 5 are served by health caregiver \#1; customers 3,6 , and 8 are served by health caregiver \#2; customers 1 and 7 are served by health caregiver \#3; the route sequence of health caregiver \#1 is $0-4$ $2-5$; the route sequence of health caregiver $\# 2$ is $0-6-8-3$; and the route sequence of health caregiver \#3 is $0-1-7$.

3.2.3. Algorithm Steps. The basic steps of the hybrid WOAPSO algorithm are as follows:

Step 1: initialize the basic parameters of the algorithm by setting the population number $P$, the league competition mechanism selection proportion $g$, the learning factor $M_{1}$, and the number of iterations Genmax.

Step 2: calculate the objective function value of each search agent, and the optimal search agent is denoted by $X^{*}(t)$, whose function value is the smallest.

Step 3: select the search mode and update the agent position using formula (16).

Step 4: assume each agent approaches the optimal search agent using formula (17) and recalculate the optimal search agent.

Step 5: assess search agents, stochastically select $g \%$ agents, and select the optimal agent for admission into the next generation until the new population scale reaches the original population scale.

Step 6: proceed to Step 7 when the maximum number of iterations or the threshold is reached; otherwise, return to Step 3 for the next search.

Step 7: terminate the algorithm and generate the objective function value of the optimal individual.

\section{Experimental Simulation and Analysis}

All algorithms are programmed using MATLAB 2018 and are tested on an Intel i7-4800MQ $(2.7 \mathrm{GHz}$ and $4 \mathrm{~GB})$ computer with a WIN7 operating system.

4.1. Example Description. To test the performance of the proposed algorithm, 10 examples are generated in each of three scales: 25 customers, 45 customers, and 60 customers. $N$ points are randomly generated within the Cartesian

\begin{tabular}{|c|c|c|c|c|c|c|c|c|}
\cline { 2 - 8 } Customer number & 1 & 2 & 3 & 4 & 5 & 6 & 7 & 8 \\
\cline { 2 - 9 } Position vector & 3.6 & 1.7 & 2.1 & 1.9 & 1.1 & 2.6 & 3.1 & 2.4 \\
\hline
\end{tabular}

Figure 2: Coding scheme of the hybrid algorithm.

Step 1: perform $[X]$ operation for each dimension of an individual.

Step 2: group customers according to $[X]$.

Step 3: perform $\{X\}$ operation in each group.

Step 4: rank according to $\{X\}$ value to obtain the route sequence.

FIGURE 3: Decoding process of the hybrid algorithm.

coordinate plane $[0,100]^{2}$, and the distance between two points is the Euclidean distance. Service time is the integer of uniformly distributed $[10,15]$. The coordinate of the health care center is $[0,0]$.The longest working time $L$ is 250 . The midpoint $L K_{i}$ and width $L L_{i}$ of the stochastic number time window are used to determine the customer time window. The midpoint $L K_{i}$ is the stochastic integer of uniformly distributed [0,250]; the width $L L_{i}$ is the stochastic integer of uniformly distributed $[10,15]$; and the tolerable time window width $T L_{i}$ is the stochastic integer of the uniformly distributed $\left[L L_{i}+30, L L_{i}+50\right]$. The unit travel cost, unit service cost, unit overtime cost, and waiting cost are $3,20,15$, and 10 , respectively.

4.2. Algorithm Performance Analysis. To verify the superiority of the proposed WOA-PSO algorithm for solving the scheduling problem of home health caregiver considering working time, a large number of simulation experiments are conducted on the proposed algorithm. The simulation experiment results are compared with those of WOA and PSO. The simulation results in Figure 4 show that WOA-PSO algorithm has a higher convergence rate than WOA and a stronger local search compared to PSO.

Three different scale cases are used to test the hybrid algorithm. They are Scale 1 (25 customers), Scale 2 (40 customers), and Scale 3 (60 customers). From Figures 5-7, the algorithm converges in the 12 th generation in a 25 -scale example, in the 39th generation in a 40 -scale example, and in the 36th generation in a 60 -scale example. It can be seen that the algorithm achieves a high convergence rate and stability in these examples of different scales.

From Tables 1 and 2, in Scale 1 example, compared with the PSO algorithm and the WOA, the obtained objective function value by WOA-PSO algorithm is decreased by $10.30 \%$ and $6.64 \%$ and the calculation time of the WOA-PSO algorithm is reduced by $33.33 \%$ and $45.71 \%$. In Scale 2 example, the obtained objective function value by WOAPSO algorithm is decreased by $4.47 \%$ and $0.81 \%$ and the calculation time of the WOA-PSO algorithm is reduced by $9.81 \%$ and $49.91 \%$. In Scale 3 example, the obtained objective function value by WOA-PSO algorithm is decreased by $2.80 \%$ and $0.40 \%$ and the calculation time of the WOA-PSO algorithm is reduced by $1.11 \%$ and $48.45 \%$. It can be seen 


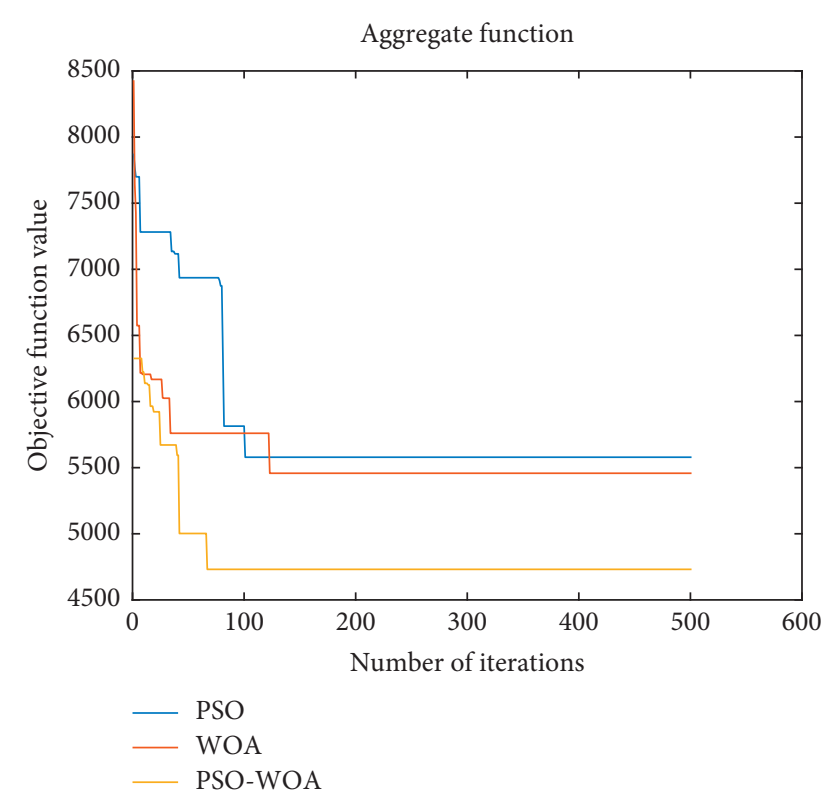

FIgUre 4: Algorithm comparisons.

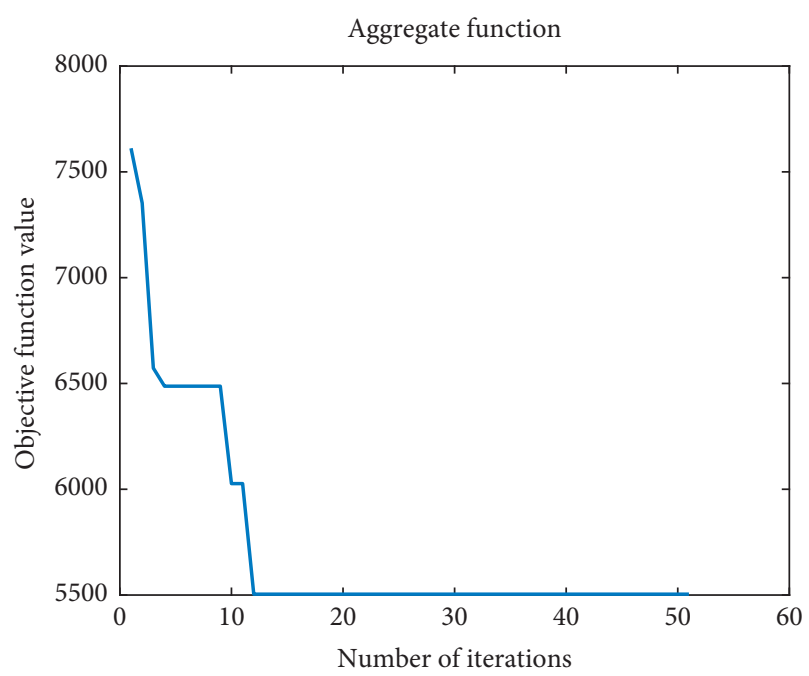

Figure 5: Scale 1 (25 customers).

that the optimization speed and optimization result of the hybrid optimization algorithm in this paper are superior to the single algorithm in the case of the same simulation environment and parameters. The results show that the hybrid algorithm is more feasible and suitable for solving such optimization problems.

4.3. Benchmark Test. To further test the performance of the WOA-PSO algorithm for solving VRPTW, the Solomon example is applied (http://web.cba.neu.edu/ msolomon/ problem.htm) as a benchmark, calculating each example 20 times. Table 3 provides the experimental results, expressed as the mean value of the optimal solution and the relative deviation between the mean value and the known optimal value for each example after 20 repeated calculations.

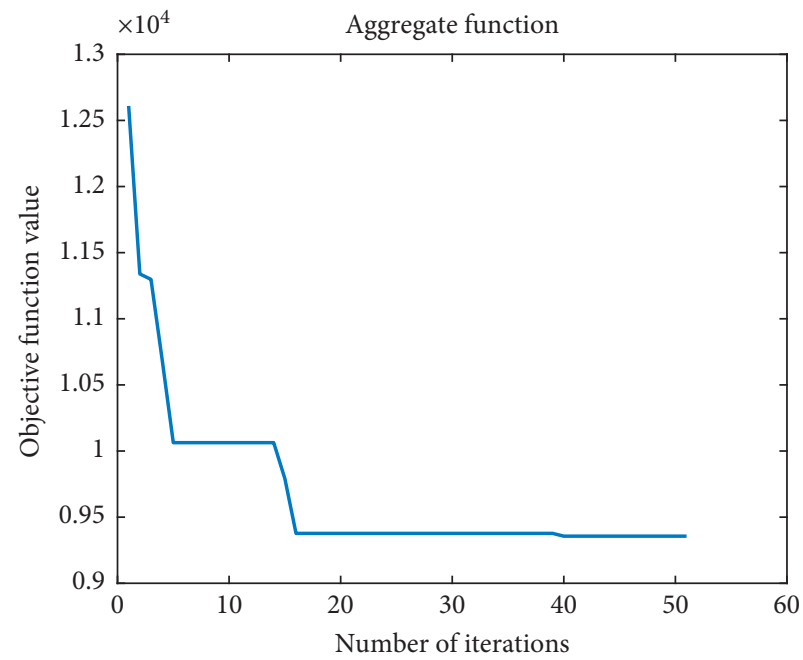

Figure 6: Scale 2 (40 customers).

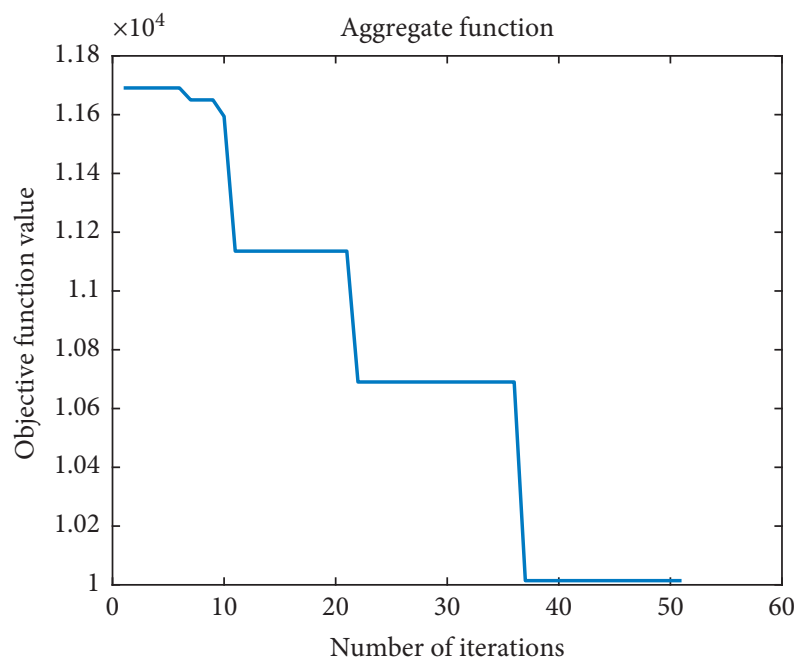

Figure 7: Scale 3 (60 customers).

TABle 1: Optimization time comparison among different algorithms.

\begin{tabular}{lccc}
\hline & \multicolumn{3}{c}{ Optimization time (s) } \\
& WOA & PSO & WOA-PSO \\
\hline Scale 1 & 4.31 & 3.51 & 2.34 \\
Scale 2 & 10.64 & 5.91 & 5.33 \\
Scale 3 & 25.84 & 13.47 & 13.32 \\
\hline
\end{tabular}

TABle 2: Optimization result comparison among different algorithms.

\begin{tabular}{lccc}
\hline & \multicolumn{3}{c}{ Results of 20 times of optimization } \\
& WOA & PSO & WOA-PSO \\
\hline Scale 1 & 5033.46 & 5239.18 & 4699.33 \\
Scale 2 & 9648.94 & 10017.98 & 9569.94 \\
Scale 3 & 11736.09 & 12025.90 & 11689.15 \\
\hline
\end{tabular}


TABLE 3: Solving the Solomon example with the WOA-PSO algorithm.

\begin{tabular}{lccc}
\hline Example & Scale & Known optimal solution & \multicolumn{2}{c}{ Results obtained by WOA-PSO } \\
Relative deviation (\%)
\end{tabular}

Table 3 shows that the relative deviation between the mean value of the WOA-PSO algorithm and the current optimal value is less than $4.5 \%$. The relative deviation between the mean value of all examples compared to the mean value of the known optimal solution is $2.34 \%$. The WOAPSO algorithm achieves excellent performance for solving VRPTW and is equivalent to the known optimal solution in each case.

The experimental results of the WOA-PSO algorithm are compared with the optimal solution obtained by the WOA-TS algorithm proposed in the literature [16] and the known optimal solution, as detailed in Table 4. The last column lists the relative deviation between the optimal solution obtained by the proposed algorithm and the known optimal solution.

Table 4 shows that, compared to the WOA-TS algorithm, the maximum optimal value of the WOA-PSO algorithm increases by $6.31 \%$ given the same number of vehicles. The relative deviation between the optimal value and the value of the known optimal solution does not exceed $2.3 \%$. Thus, the WOA-PSO algorithm fully uses the global search ability of PSO. It also relies on a competitive mechanism and dualdomain structure to effectively enhance local search ability, avoid being caught in local optimization too early, and improve solution quality.

4.4. Experimental Analysis of Large-Scale Examples. As the demand for pension increases, the number of customers served by home care medical enterprises will increase, so large-scale examples are used to verify the WOA-PSO algorithm proposed in this paper. Ten cases of 100 customers and ten cases of 200 customers are generated randomly. Each case is run 10 times, and the average value is recorded. For large-scale examples, the running time of the heuristic algorithm is long, so all algorithms are run in the same running time to ensure the validity of the experiments. The experimental results are shown in Table 5.

The running results are shown in Table 5. For all the scale 100 examples, 90\% cases' running results with WOAPSO are better than comparison algorithm, and the optimization range is between $9 \%$ and $13 \%$. For all the scale 200 examples, $70 \%$ cases' running results with WOA-PSO are better than comparison algorithm, but the optimization range is between $7 \%$ and $9 \%$. The main reason for the phenomenon is that the running speed of the algorithm slows down with the increase of the example size. Therefore, WOA-PSO cannot fully find a better solution in the case of the same running time for the scale 200 example.

4.5. Cost Analysis. The research on the home health caregiver scheduling in the resource sharing mode can resolve the contradiction between scarce care worker resources and huge demand, improve customer satisfaction, and reduce the cost of home care center. Therefore, the cost structure and customer satisfaction of health care centers are selected as indicators in the experimental section. Taking E25-3 as an example, the resource sharing mode and the traditional mode are compared from five aspects: waiting cost, overtime cost, travel cost, service cost, and customer satisfaction, as shown in Figure 8 and Table 6.

Figure 8 shows that the resource sharing mode reduces the total cost by $9.5 \%$ and lowers both the service cost and waiting cost by $25.16 \%$ and $26.35 \%$, respectively, compared to the traditional mode, while travel cost is increased by $4.35 \%$ and overtime cost is increased by $34.25 \%$. There are two explanations for this: first, in order to match the working time with the customer's expected time, health caregivers must choose customers who are located far away, increasing travel cost; second, given the limitations in working time, 
TABLE 4: Comparison between the WOA-PSO algorithm and existing algorithms.

\begin{tabular}{|c|c|c|c|c|c|}
\hline Example & Scale & $\begin{array}{l}\text { WOA-TS } \\
\text { Optimal route }\end{array}$ & $\begin{array}{l}\text { WOA-PSO } \\
\text { Optimal route }\end{array}$ & $\begin{array}{c}\text { Known optimal solution } \\
\text { Optimal route }\end{array}$ & Relative deviation (\%) \\
\hline $\mathrm{C} 104$ & 25 & 187.5 & 187.5 & 186.9 & 0.32 \\
\hline $\mathrm{C} 201$ & 25 & 214.1 & 214.1 & 214.7 & -0.28 \\
\hline R103 & 25 & 455.7 & 455.7 & 454.6 & 0.24 \\
\hline R201 & 25 & 467.7 & 464.4 & 463.3 & 0.24 \\
\hline $\mathrm{RC} 101$ & 25 & 466.7 & 462.2 & 461.1 & 0.24 \\
\hline RC208 & 25 & 271.5 & 271.5 & 269.1 & 0.89 \\
\hline $\mathrm{C} 103$ & 50 & 439.9 & 362.2 & 361.4 & 0.22 \\
\hline C201 & 50 & 373.8 & 361.2 & 360.2 & 0.28 \\
\hline $\mathrm{R} 101$ & 50 & 1081.2 & 1046.7 & 1044 & 0.26 \\
\hline R201 & 50 & 831.6 & 794.3 & 791.9 & 0.30 \\
\hline $\mathrm{RC} 101$ & 50 & 988.4 & 951.6 & 944 & 0.51 \\
\hline RC204 & 50 & 459.4 & 449.2 & 444.2 & 1.13 \\
\hline $\mathrm{C} 101$ & 100 & 1273.6 & 828.9 & 827.3 & 0.19 \\
\hline C201 & 100 & 719.3 & 597.9 & 589.1 & 0.47 \\
\hline R103 & 100 & 1284 & 1235.5 & 1208.7 & 2.22 \\
\hline $\mathrm{RC} 101$ & 100 & 1698 & 1631.8 & 1619.8 & 0.74 \\
\hline RC205 & 100 & 1221.6 & 1162.1 & 1154 & 0.70 \\
\hline
\end{tabular}

TABle 5: Test results of large-scale examples.

\begin{tabular}{|c|c|c|c|c|c|c|c|}
\hline Case no. & WOA-PSO & $\mathrm{PSO}$ & WOA & Case no. & WOA-PAO & $\mathrm{PSO}$ & WOA \\
\hline 100_1 & 9521.66 & 9710.41 & 9637.42 & 200_1 & 11503.26 & 11720.96 & 11590.73 \\
\hline 100_2 & 8495.22 & 8601.37 & 8556.79 & 200_2 & 14724.42 & 14637.68 & 14534.98 \\
\hline $100 \_3$ & 11034.71 & 11160.63 & 10978.62 & 200_3 & 15800.42 & 15724.22 & 15547.39 \\
\hline 100_4 & 15314.49 & 15683.85 & 15366.38 & 200_4 & 16468.44 & 16754.65 & 16539.50 \\
\hline $100 \_5$ & 13457.68 & 13067.32 & 12877.72 & $200 \_5$ & 15826.45 & 16163.20 & 16037.51 \\
\hline 100_6 & 19865.48 & 20122.66 & 19958.18 & 200_6 & 16681.47 & 16944.54 & 16781.46 \\
\hline 100_7 & 16503.37 & 16711.56 & 16491.84 & 200_7 & 24236.40 & 24349.94 & 24138.60 \\
\hline $100 \_8$ & 12112.59 & 12467.36 & 12239.09 & 200_8 & 26984.36 & 27338.86 & 27121.38 \\
\hline $100 \_9$ & 11163.22 & 10932.61 & 111336.78 & 200_9 & 17721.60 & 17503.46 & 17829.37 \\
\hline 100_10 & 12946.97 & 13168.34 & 13011.28 & 200_10 & 17406.58 & 17221.50 & 17104.00 \\
\hline
\end{tabular}

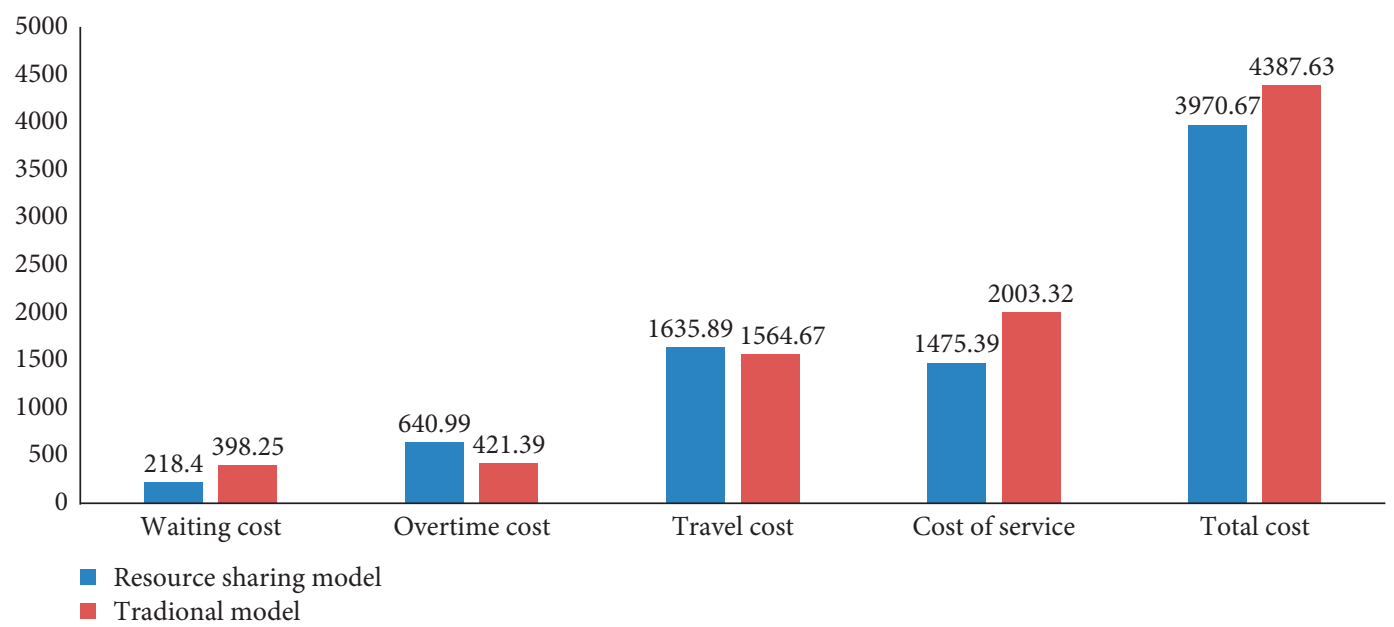

Figure 8: Cost comparison.

they must work overtime to meet the requirements of all customers in the area, increasing overtime cost. Table 6 shows that the customer satisfaction on the three scale examples is increased by $14.03 \%, 15.25 \%$, and $18.6 \%$ in the resource sharing model, respectively. This may be because health caregivers usually choose customers whose schedules can be met in the first place. Thus, adopting the resource sharing model for home health caregiver scheduling can 
TABLE 6: Customer satisfaction comparison.

\begin{tabular}{lcc}
\hline & \multicolumn{2}{c}{ Customer satisfaction } \\
& Resource sharing mode & Traditional mode \\
\hline Scale 1 & 14.67 & 12.19 \\
Scale 2 & 20.84 & 17.66 \\
Scale 3 & 26.44 & 21.52 \\
\hline
\end{tabular}

reduce the operational cost of health caregivers, save human resources, and improve service quality.

\section{Conclusions}

This paper investigates the home health caregiver scheduling problem under a resource sharing model. A corresponding mathematical model is established and the WOA-PSO hybrid algorithm is proposed to solve it. The basic WOA has poor convergence performance and is prone to fall into a local optimum. Therefore, the league competition mechanism and the PSO learning mechanism in the WOA are embedded to deal with the subswarms, which make the hybrid algorithm identify an optimization scheme efficiently and effectively. The hybrid WOA-PSO algorithm proposed in this paper can quickly solve the examples on three scales (i.e., 25, 40, and 60 customers). A benchmark test on the Solomon example verifies its effectiveness. Moreover, the comparison results between the resource sharing model and the traditional model from cost structure and customer satisfaction highlight the superiority of the resource sharing model. The resource sharing model can satisfactorily resolve the contradiction between insufficient medical resources and serious social aging in China and can better allocate human resources and can effectively reduce social costs. However, the scheduling problem of domestic home health resources is a complex system. Therefore, how to use the multiagent systems [21] to describe the model will be our future research interest.

\section{Data Availability}

The data used to support the findings of this study are included within the article. Part of the data is generated randomly according to the problem and part of the data is from Solomon example.

\section{Conflicts of Interest}

The authors declare that they have no conflicts of interest.

\section{Acknowledgments}

This study was supported by the National Natural Science Foundation of China (71872002), Humanities and Social Sciences Research Project of Ministry of Education of China (Funding No. 19YJCZH091), and Humanities and Social Sciences Research Project for University in Anhui (Funding No. SK2017A0077).

\section{References}

[1] D. S. Mankowska, F. Meisel, and C. Bierwirth, "The home health care routing and scheduling problem with interdependent services," Health Care Management Science, vol. 17, no. 1, pp. 15-30, 2014.

[2] National Bureau of Statistics, China Health Statistics Yearbook 2018, China Statistics Publishing House, Beijing, China, 2018.

[3] R. Redjem, S. Kharraja, X. Xie et al., "Coordinated multicriteria scheduling of caregivers in home health care services," in Proceedings of the IEEE Conference on Automation Science and Engineering, CASE 2011, Trieste, Italy, August 2011.

[4] M. S. Rasmussen, T. Justesen, A. Dohn, and J. Larsen, “The home care crew scheduling problem: preference-based visit clustering and temporal dependencies," European Journal of Operational Research, vol. 219, no. 3, pp. 598-610, 2012.

[5] J. Larsen, M. Chaieb, and K. Mellouli, "The home care scheduling problem: a modeling and solving issue," in Proceedings of the International Conference on Modeling, Berkeley, CA, USA, August 2013.

[6] K. Braekers, R. F. Hartl, S. N. Parragh, and F. Tricoire, "A biobjective home care scheduling problem: analyzing the tradeoff between costs and client inconvenience," European Journal of Operational Research, vol. 248, no. 2, pp. 428-443, 2016.

[7] S. Tricoire, A. Matta, E. Sahin et al., "A two-stage approach for solving assignment and routing problems in home health care services," in Proceedings of the International Conference on Health Care Systems Engineering, pp. 66-79, Copenhagen, Denmark, May 2013.

[8] R. Liu, B. Yuan, and Z. Jiang, "Mathematical model and exact algorithm for the home care worker scheduling and routing problem with lunch break requirements," International Journal of Production Research, vol. 55, no. 2, pp. 558-575, 2016.

[9] Y. Zhuo, R. Liu, and Y. Hua, "Taboo solving algorithms for periodic home-based medical nursing problems," Chinese Science and Technology Paper, vol. 10, no. 14, pp. 1714-1720, 2015.

[10] T. Yang-yi, R. Liu, and Z. Jiang, "Scheduling of family nurses with simultaneous service demand," Industrial Engineering and Management, vol. 22, no. 3, 2017.

[11] B. Yuan, R. Liu, and Z. Jiang, "Study on scheduling problems of multi-type family nursing staff," Journal of Systems Engineering, vol. 32, no. 1, pp. 57-64, 2017.

[12] L. Fang, L. Ma, S. Ding, and D. Zhao, "Finite-time stabilization for a class of high-order stochastic nonlinear systems with an output constraint," Applied Mathematics and Computation, vol. 358, pp. 63-79, 2019.

[13] S. H. Zhao, J. H. Park, and C. C. Chen, "Second-order sliding mode controller design with output constraint," Automatica, vol. 112, Article ID 108704, 2020.

[14] X. Liang, J. Xia, G. Chen et al., "Dissipativity-based nonfragile sampled-data control for fuzzy Markovian jump systems," International Journal of Fuzzy Systems, vol. 21, no. 12, 2019.

[15] J. Xia, J. Zhang, J. Feng, Z. Wang, and G. Zhuang, "Command filter-based adaptive fuzzy control for nonlinear systems with unknown control directions," IEEE Transactions on Systems, Man, and Cybernetics: Systems, vol. 29, pp. 1-9, 2019.

[16] J. Yan-ning, X. Qi, H. Ren et al., "Automobile logistics routing problem optimization under resource sharing mode," Journal of Highway and Transportation Research and Development, vol. 34, no. 6, pp. 114-121, 2017. 
[17] S. A. AlQahtani, "An efficient resource allocation to improve QoS of 5G slicing networks using general processor sharingbased scheduling algorithm," International Journal of Communication Systems, vol. 33, no. 4, 2019.

[18] L. Wu, X. Meng, S. Liu et al., "A trading supported manufacturing resource sharing model for manufacturing grid," in Proceedings of the Ninth International Conference on Computer Supported Cooperative Work in Design, Coventry, UK, May 2005.

[19] D. Wu, J. Wang, R. Q. Hu, Y. Cai, and L. Zhou, "Energyefficient resource sharing for mobile device-to-device multimedia communications," IEEE Transactions on Vehicular Technology, vol. 63, no. 5, pp. 2093-2103, 2014.

[20] S. Mirjalili and A. Lewis, "The whale optimization algorithm," Advances in Engineering Software, vol. 95, pp. 51-67, 2016.

[21] S. Huo and Y. Zhang, " $H_{\infty}$ consensus of Markovian jump multi-agent systems under multi-channel transmission via output feedback control strategy," ISA Transactions, vol. 99, pp. 28-36, 2020. 